\title{
Do lip bumpers work?
}

\author{
Abstracted from \\ Hashish DI, Mostafa YA.
}

Effect of lip bumpers on mandibular arch dimensions. Am J Orthod Dentofacial Orthop 2009; 135: 106-109

Address for correspondence: Yehya A Mostafa, Department of Orthodontics and Dentofacial Orthopaedics, Cairo University, Faculty of Dentistry, PO Box 60, Mina Garden Post Office, October City, Cairo 12582, Egypt. E-mail: mangoury@usa.net

\section{Question: What are the effects of the lip bumper on mandibular arch dimensions in adolescents?}

Data sources Searches were made using PubMed, Embase, the Cochrane Central Register of Controlled Trials and the Cochrane database of systematic reviews, along with searches by hand of the following journals: Angle Orthodontist, American Journal of Orthodontics and Dentofacial Orthopedics, Journal of Orthodontics and European Journal of Orthodontics.

Study selection The first step was to identify eligible reports based on their titles and abstracts: 52 articles were found. Inclusion and exclusion criteria were then applied. Studies were included if subjects were human; they were randomised controlled trials and prospective or retrospective studies; they discussed the effect of the lip bumper on the arch and teeth; and they were reported in the English language. Articles excluded were mainly animal studies, case reports, case series, review articles, abstracts, in-vitro studies, discussions and interviews; were published in a language other than English; or did not follow the objective of this review. The selection was made by two researchers. Their results were compared to identify discrepancies and reach mutual agreement. Data extraction and synthesis If two or more studies had evaluated the same technique, a meta-analysis was planned.

Results A total of 16 RCT were identified of which 13 underwent detailed evaluation; of these, only one study was considered to have usable outcome information. The others were excluded because of control group, sex, age, study casts and cephalometric analysis, and measurement error. This single small study showed that lip bumper can increase arch dimensions and contribute to crowding relief in mixed dentition.

Conclusions The results showed increases in arch dimensions, including a greater arch length. This was attributed to incisor proclination, distalisation, and distal tipping of the molars. There were also increases in arch width and intercanine and deciduous intermolar or premolar distances. The long-term stability of the effects of the lip bumper need to be elucidated.

\section{Commentary}

The decision whether or not to extract teeth in cases where there is crowding has always been, and will continue to be, one of the most contentious issues in orthodontics. There is no doubt that for the orthodontist, and particularly for the patient, nonextraction therapy is extremely attractive. As well as obviating the need for the removal of (often sound) teeth, treatment times are significantly shorter with nonextraction therapy as orthodontists often spend a number of months closing residual spaces. Apart from interdental stripping, which involves the loss of a limited amount of dental enamel, space can be gained in the lower arch either by maintenance of the leeway space at the end of the mixed dentition, or by expansion of the arch. Expansion can be anteroposterior (with proclination of the incisors or distal movement of the lower molars), or transverse, with an increase in arch width, or a combination of these.

This review article examines the effects of lip bumper therapy on mandibular arch dimensions. Lip bumpers consist of a single, thick, stainless steel wire running buccally on the outside of the lower arch from the lower left first molar to the lower right first molar. They often incorporate a small acrylic shield in the lower incisor area. They are usually removable, with a U-loop and terminal lug inserting into a tube on a molar band. Progressive advancement of the labial portion can thus be carried out by activation of the loop.

This paper aimed to investigate the effects of lip bumpers on mandibular arch dimensions in adolescents compared with untreated patients. Fifty-two studies published between 1968 and 2007 were identified and, of these, 16 met the inclusion criteria. Fifteen were subsequently rejected because of methodological errors and only one study, carried out by Davidovich and colleagues, ${ }^{1}$ was finally selected. This study, although a prospective controlled clinical trial, had only 16 subjects in the experimental group and 18 in the control group. Despite accepting only this study, the effects of lip bumpers on mandibular arch dimensions were discussed with reference to numerous other articles which had failed the inclusion criteria.

The effects were discussed under three headings: molar distalisation, arch width and incisor proclination. A number of the studies showed significantly more molar distalisation with lip bumpers than in untreated cases. There is therefore some evidence that not only do lip bumpers maintain arch length, thus preserving leeway space, but (particularly if advanced) distalisation of the lower first molars will take place. The present article did not discuss one potential problem that this may create in the mixed dentition: the effect on the lower second permanent molars. Where there is crowding in the lower arch, particularly as a result of dento-alveolar discrepancy, this is not confined to the anterior teeth and thus lower second permanent molar impaction may ensue.

Transverse arch dimensions were also found to increase with lip bumper therapy. Davidovich's study ${ }^{1}$ showed a small increase in the width between the second deciduous molars as well as the perma- 
nent canines, but a number of other studies investigating lip bumper therapy in the full adult dentition showed the largest increase between the first and second premolars. ${ }^{2}$

Incisor proclination was significantly higher in treated subjects than in the untreated group. Other studies quoted also reported significant incisor proclination. It must be remembered that for most malocclusions, significant proclination of the lower incisors outside the zone of neutral balance is potentially unstable. Over time, this has a strong tendency to relapse, which may result in an increase in the overjet and/ or an increase in lower labial segment crowding. Indeed, there is strong evidence that increasing lower intermolar and intercanine widths during treatment has a high relapse tendency.

In their conclusion, the authors do comment that the long-term effects of lip bumper therapy need to be elucidated.

Julian O'Neill

Orthodontic Department, Kettering General Hospital NHS Trust, Kettering, Northamptonshire, UK

\section{Practice points}

- Lip bumpers may be useful in the following situations: space maintenance and preservation of the leeway space in cases in the late mixed dentition where there is mild crowding; or as an aid to space creation in cases with mild lower arch crowding, especially where this is not because of true dento-alveolar discrepancy (such as early loss of lower deciduous molars with some mesial drift of lower first permanent molars.)

- Caution should be exercised where significant distal molar movement or incisor proclination becomes evident. An orthopantomogram (OPT) radiograph will indicate the amount of space available for eruption of lower second permanent molars.

1. Davidovitch $M$, Mclnnis $D$, Lindauer SJ. The effect of lip bumper therapy in mixed dentition. Am J Orthod Dentofacial Orthop 1997; 111: 52-58.

2. Burke SP, Silveira AM, Goldsmith LI, Yancey JM, Van Stewart A, Scarfe WC.

A meta-analysis of mandibular intercanine width in treatment and postretention. Angle Orthod. 1998; 68: 53-60.

Evidence-Based Dentistry (2009) 10, 48-49. doi:10.1038/sj.ebd.6400651 